\title{
Monogamy of quantum discord
}

\author{
Yu Guo \\ Institute of Quantum Information Science, School of Mathematics and Statistics, \\ Shanxi Datong University, Datong, Shanxi 037009, China \\ E-mail: guoyu3@aliyun.com
}

\section{Lizhong Huang}

Institute of Quantum Information Science, School of Mathematics and Statistics, Shanxi Datong University, Datong, Shanxi 037009, China

\section{Yang Zhang}

Institute of Theoretical Physics, Shanxi Datong University, Datong 037009, China

\begin{abstract}
The original quantum discord (QD) is shown to be not monogamous except for the three-qubit states. Recently, a complete monogamy relation for multiparty quantum system was established for entanglement in [Phys. Rev. A. 101, 032301 (2020)], and in addition, a new multipartite generalization of QD was proposed in [Phys. Rev. Lett. 124, 110401 (2020)]. In this work, we firstly define the complete monogamy for this multipartite quantum discord (MQD) and the global quantum discord (GQD). MQD, with the same spirit as the complete monogamy of entanglement, is said to be completely monogamous (i) if it does not increase under coarsening of subsystems and (ii) if some given combination of subsystems reach the total amount of the correlation, then all other combination of subsystems that do not include all the given subsystems do not contain such a correlation any more. Here, coarsening of subsystems means discarding or combining some subsystems up to the given partition. Simultaneously, the complete monogamy of GQD is also defined with slight modification on the coarsening relation. Consequently, we explore all the coarsening relations of MQD and show that it is completely monogamous with a modicum assumption. In addition, with the same spirit, we investigate all the coarsening relations for GQD and show that GQD is not completely monogamous. That is, in the sense of the complete monogamy relation, MQD as a generalization of the original QD captures the nature of such a quantum correlation, and thus it is nicer than GQD as a generalization.
\end{abstract}

Keywords: Quantum discord, Multipartite state, Complete monogamy relation, Coarsening relation 


\section{Introduction}

Quantum discord, as the foremost one of the quantum correlations beyond entanglement, has been extensively explored in the last two decades due to its remarkable applications in quantum information protocols $[1,2,3,4,5,6,7,8,9,10,11,12,13]$. It is originally defined for bipartite system as the minimized difference between the quantum mutual information with and without a von Neumann projective measurement applied on one of the subsystems [1, 2]. Consequently, several multipartite generalizations have been proposed based on different scenarios $[7,8,11]$. In Ref. [7], by means of the multipartite mutual information, the global quantum discord is defined as the minimal difference between the mutual information the pre- and post-state under local von Neumann measurements. In Ref. [8], the total quantum discord and the genuine quantum discord were investigated based on the total information and the total classical correlation. Very recently, Radhakrishnan et al. [11] gave another way of extending quantum discord to multipartite case up to a fixed conditional local von Neumann measurement. The global quantum discord, total quantum discord and the genuine quantum discord are symmetric under exchange the subsystems, while the multipartite quantum discord in Ref. [11] is asymmetric.

An important issue closely related to a nonlocal correlation measure of composite quantum system (such as entanglement measure, quantum discord, and EinsteinPodolsky-Rosen steering, etc.) is to explore the distribution of the correlation over many parties $[14,15,16,17,18,19,20,21,22]$. In this context, there are two ways to describe the distribution: one is the monogamy or polygamy relation by the bipartite

measure $[14,15,16,17,18]$ and the other one is the relation based on the multipartite measure [19, 20, 23]. Among these attempts, numerous efforts have been made for entanglement. However, there are very few studies on quantum discord. For the bipartite measure of quantum discord, it is proved that the square of quantum discord obeys the monogamy relation for three qubit states [24] while other systems always display polygamous behavior [24, 25, 26, 27, 28]. In Ref. [23], it has been shown that global quantum discord acts as a monogamy bound for pairwise quantum discord in which one of the subsystem is fixed while the other part runs over all subsystems provided that bipartite discord does not increase under discard of subsystems. According to the framework of complete monogamy relation (for entanglement), when we deal with the multipartite systems, the traditional approach of exploring monogamy relation based the bipartite measure is not substantial. There are something missing [19]. Therefore, for multipartite quantum discord and global quantum discord, we need to examine renewedly with the complete monogamy protocol.

The aim of this article is to explore the distribution of discord contained in a multipartite state whenever it is measured by multipartite quantum discord and global quantum discord respectively in the framework of the complete monogamy relation. However, quantum discord is far different from entanglement: quantum discord is not monotonic under local operation and classical communication (LOCC) while it is a basic 
feature for entanglement. So we need at first to consider the coarsening relations when we consider monogamy of multipartite quantum discord and global quantum discord and establish the framework of complete monogamy relation for multipartite quantum discord and global quantum discord.

The rest of this paper is organized as follows. We recall the definitions of the original quantum discord, multipartite quantum discord, global quantum discord, and investigate coarser relation of multiparty partitions by which we then introduce the monogamy laws in literatures in Section II. In Section III, we put forward the definition of complete multiparty quantum discord and by which we discuss and establish the framework of complete monogamy relation for multipartite quantum discord and global quantum discord. Section IV explores the coarsening relation and complete monogamy relation of the multipartite quantum discord. It is divided into three subsections. The first subsection discusses the tripartite case, the second subsection deals with the fourpartite case and the main conclusioin is given in the last subsection for the general multipartite case. In Section V, we deal with global quantum discord in the framework of complete monogamy relation. Finally, we conclude. For convenience, throughout this paper, we call the $n$-partite discord in Ref. [11] multipartite quantum discord (MQD), and the one in Ref. [7, 23] global quantum discord (GQD), and we call both MQD and GQD multiparty quantum discord in order to differentiate these different concepts.

\section{Preliminaries}

In this section, we review at first the definitions of MQD and GQD, and then recall the monogamy relation and complete monogamy relation in literatures. Throughout this paper, we let $\mathcal{H}^{A_{1} A_{2} \cdots A_{n}}$ be the Hilbert space corresponding to the $n$-parite quantum system with finite dimension. and let $\mathcal{S}^{X}$ be the set of density operators acting on $\mathcal{H}^{X}$. The tripartite and the four-partite systems are always denoted by $\mathcal{H}^{A B C}$ and $\mathcal{H}^{A B C D}$, respectively.

\subsection{Quantum discord and its generalization}

For any bipartite state $\rho \in \mathcal{S}^{A B}$, the original quantum discord is defined as $[1,2]$

$$
D_{A ; B}(\rho)=\min _{\Pi^{A}}\left[S_{B \mid \Pi^{A}}(\rho)-S_{B \mid A}(\rho)\right]
$$

where $S_{B \mid A}(\rho)=S_{A B}(\rho)-S_{A}(\rho), S(\rho)=-\operatorname{Tr} \rho \log \rho$ is the von Neumann entropy,

$$
S_{B \mid \Pi^{A}}(\rho)=\sum_{j} p_{j}^{A} S_{A B}\left(\Pi_{j}^{A} \rho \Pi_{j}^{A} / p_{j}^{A}\right)=S_{A B}\left(\rho_{\Pi^{A}}\right)-S_{A}\left(\rho_{\Pi^{A}}\right),
$$

$\Pi_{j}^{A}$ is a one-dimensional von Neumann projection operator on subsystem $A$ and

$p_{j}^{A}=\operatorname{Tr}\left(\Pi_{j}^{A} \rho \Pi_{j}^{A}\right), \rho_{\Pi^{A}}=\sum_{j} \Pi_{j}^{A} \rho \Pi_{j}^{A}$. Hereafter, we denote $S\left(\rho^{X}\right)$ by $S_{X}(\rho)$ sometimes for simplicity.

For multipartite systems, the $(n-1)$-partite measurement is written [11]

$$
\Pi_{j_{1} \ldots j_{n-1}}^{A_{1} \ldots A_{n-1}}=\Pi_{j_{1}}^{A_{1}} \otimes \Pi_{j_{2} \mid j_{1}}^{A_{2}} \cdots \otimes \prod_{j_{n-1} \mid j_{1} \ldots j_{n-2}}^{A_{n-1}},
$$


where the $n$ subsystems are labeled as $A_{i}$, the measurements take place in the order $A_{1} \rightarrow A_{2} \rightarrow \ldots A_{n-1}$. For $\rho \in \mathcal{S}^{A_{1} A_{2} \cdots A_{n}}$, the $n$-partite MQD is defined by [1]]

$$
\begin{aligned}
D_{A_{1} ; A_{2} ; \ldots ; A_{n}}(\rho)= & \min _{\Pi^{A_{1} \ldots A_{n-1}}}\left[-S_{A_{2} \ldots A_{n} \mid A_{1}}(\rho)+S_{A_{2} \mid \Pi^{A_{1}}}(\rho) \ldots\right. \\
& \left.+S_{A_{n} \mid \Pi^{A_{1} \ldots A_{n-1}}}(\rho)\right]
\end{aligned}
$$

up to the measurement ordering $A_{1} \rightarrow A_{2} \rightarrow \ldots \rightarrow A_{n-1}$, where

$$
S_{A_{k} \mid \Pi^{A_{1} \ldots A_{k-1}}}(\rho)=\sum_{j_{1} \ldots j_{k-1}} p_{j}^{(k-1)} S_{A_{1} \ldots A_{k}}\left(\Pi_{j}^{(k-1)} \rho \Pi_{j}^{(k-1)} / p_{j}^{(k-1)}\right)
$$

with $\Pi_{j}^{(k)} \equiv \Pi_{j_{1} \ldots j_{k}}^{A_{1} \ldots A_{k}}, p_{j}^{(k)}=\operatorname{Tr}\left(\Pi_{j}^{(k)} \rho \Pi_{j}^{(k)}\right)$. For example, the triparite case $\rho \in \mathcal{S}^{A B C}$,

$$
\begin{aligned}
D_{A ; B ; C}(\rho) & =\min _{\Pi^{A B}}\left[-S_{B C \mid A}(\rho)+S_{B \mid \Pi^{A}}(\rho)+S_{C \mid \Pi^{A B}}(\rho)\right] \\
& =\min _{\Pi^{A B}}\left[S\left(\rho^{\prime A B}\right)-S\left(\rho^{\prime A}\right)+S\left(\rho^{\prime \prime}\right)-S\left(\rho^{\prime \prime A B}\right)-S(\rho)+S\left(\rho^{A}\right)\right]
\end{aligned}
$$

up to the measurement ordering $A \rightarrow B$, where $\Pi^{A}(\rho)=\rho^{\prime}, \Pi^{A B}(\rho)=\rho^{\prime \prime}$, and $\rho^{X}=\operatorname{Tr}_{\bar{X}}(\rho)$ with $\bar{X}$ denotes the complementary of $X$ in $\{A, B, C\}\left(\right.$ e.g, $\left.\rho^{A B}=\operatorname{Tr}_{C}(\rho)\right)$.

The GQD, denoted by $D_{A_{1}: \cdots: A_{n}}$, for an arbitrary state $\rho \in \mathcal{S}^{A_{1} \cdots A_{n}}$ under a set of local measurements $\left\{\Pi_{j_{1}}^{A_{1}} \otimes \cdots \otimes \Pi_{j_{n}}^{A_{n}}\right\}$ is defined as $[7,23]$

$$
D_{A_{1}: \cdots: A_{n}}(\rho)=\min _{\Phi}[I(\rho)-I(\Phi(\rho))]
$$

where

$$
\Phi(\rho)=\sum_{k} \Pi_{k} \rho \Pi_{k}
$$

with $\Pi_{k}=\Pi_{j_{1}}^{A_{1}} \otimes \cdots \otimes \Pi_{j_{n}}^{A_{n}}$ and $k$ denoting the index string $\left(j_{1} \cdots j_{n}\right)$, the mutual information $I(\rho)$ is defined by $[29]$

$$
I(\rho)=\sum_{k=1}^{n} S_{A_{k}}(\rho)-S_{A_{1} \cdots A_{n}}(\rho)
$$

where

$$
\Phi\left(\rho^{A_{k}}\right)=\sum_{k^{\prime}} \Pi_{k^{\prime}}^{A_{k}} \rho^{A_{k}} \Pi_{k^{\prime}}^{A_{k}} .
$$

Note here that, the difference between $D_{A_{1} ; A_{2} ; \ldots ; A_{n}}$ and $D_{A_{1}: \cdots: A_{n}}$ is that the former is semicolon in the subscript $A_{1} ; A_{2} ; \ldots ; A_{n}$ while the latter one is with colon in the subscript $A_{1}: \cdots: A_{n}$.

\subsection{Coarser relation of multipartite partition}

For any partition $X_{1}\left|X_{2}\right| \cdots \mid X_{k}$ of $A_{1} A_{2} \cdots A_{n}$ with $X_{s}=A_{s(1)} A_{s(2)} \cdots A_{s(f(s))}, s(i)<$ $s(j)$ whenever $i<j$, and $s(p)<t(q)$ whenever $s<t$ for any possible $p$ and $q, 1 \leq s, t \leq k$. For instance, partition $A B|C| D E$ is a 3-partition of $A B C D E$. Let $X_{1}\left|X_{2}\right| \cdots \mid X_{k}$ and $Y_{1}\left|Y_{2}\right| \cdots \mid Y_{l}$ be two partitions of $A_{1} A_{2} \cdots A_{n}$ or subsystem of $A_{1} A_{2} \cdots A_{n} . Y_{1}\left|Y_{2}\right| \cdots \mid Y_{l}$ is said to be coarser than $X_{1}\left|X_{2}\right| \cdots \mid X_{k}$, denoted by

$$
X_{1}\left|X_{2}\right| \cdots\left|X_{k} \succ Y_{1}\right| Y_{2}|\cdots| Y_{l}
$$


if $Y_{1}\left|Y_{2}\right| \cdots \mid Y_{l}$ can be obtained from $X_{1}\left|X_{2}\right| \cdots \mid X_{k}$ by some or all of the following ways:

- (C1) Discarding some subsystem(s) of $X_{1}\left|X_{2}\right| \cdots \mid X_{k}$;

- (C2) Combining some subsystems of $X_{1}\left|X_{2}\right| \cdots \mid X_{k}$;

- (C3) Discarding some subsystem(s) of the last subsystem $X_{k}$ provided that the last subsystem $X_{k}$ in $X_{1}\left|X_{2}\right| \cdots \mid X_{k}$ is $X_{k}=A_{k(1)} A_{k(2)} \cdots A_{k(f(k))}$ with $f(k) \geq 2$.

For example, $A|B| C|D| E \succ A|B| C|D E \succ A| B|C| D \succ A B|C| D \succ A B \mid C D$, $A|B| C|D E \succ A| B \mid D E$. Clearly, $X_{1}\left|X_{2}\right| \cdots\left|X_{k} \succ Y_{1}\right| Y_{2}|\cdots| Y_{l}$ and $Y_{1}\left|Y_{2}\right| \cdots \mid Y_{l} \succ$ $Z_{1}\left|Z_{2}\right| \cdots \mid Z_{s}$ imply $X_{1}\left|X_{2}\right| \cdots\left|X_{k} \succ Z_{1}\right| Z_{2}|\cdots| Z_{s}$. For any partition $X_{1}\left|X_{2}\right| \cdots \mid X_{k}$ of $A_{1} A_{2} \cdots A_{n}$ or subsystem of $A_{1} A_{2} \cdots A_{n}$, the assciated MQD is $D_{X_{1} ; X_{2} ; \cdots ; X_{k}}$ up to the measurement $\Pi^{X_{1} \cdots X_{k-1}}$. For example, for partition $A|B| C D$ of $A B C D$, the associated MQD is $D_{A ; B ; C D}\left(\rho^{A B C D}\right)$ up to measurement $\Pi^{A \mid B}$, while for partition $A B \mid C$ of the subsystem $A B C$ in $A B C D$, the associated MQD is $D_{A B ; C}\left(\rho^{A B C}\right)$ up to measurement $\Pi^{A B}$, where $\Pi^{A B}$ is a von Neumann measurement acting on $A B$ with $A B$ is regarded as a sigle particle, $\rho^{A B C}=\operatorname{Tr}_{D}\left(\rho^{A B C D}\right)$. One can easily see that $X_{1}\left|X_{2}\right| \cdots\left|X_{k} \succ Y_{1}\right| Y_{2}|\cdots| Y_{l}$ if and only if $\Pi^{Y_{1} \ldots Y_{l-1}}$ can be induced by $\Pi^{X_{1} \ldots X_{k-1}}$ but not vice versa. In such a case, we say $\Pi^{Y_{1} \ldots Y_{l-1}}$ and $\Pi^{X_{1} \ldots X_{k-1}}$ are compatible. Otherwsie, it is uncompatible. For example, $\Pi^{A \mid B C}$ and $\Pi^{A \mid B}$ are uncompatible on the system $\mathcal{H}^{A B C D}$ since the projection on part $B C$ (regared as a single part) is not ncessarily a projection on $B \mid C$.

Furthermore, if $X_{1}\left|X_{2}\right| \cdots\left|X_{k} \succ Y_{1}\right| Y_{2}|\cdots| Y_{l}$, we denote by $\Xi\left(X_{1}\left|X_{2}\right| \cdots \mid X_{k}-\right.$ $\left.Y_{1}\left|Y_{2}\right| \cdots \mid Y_{l}\right)$ the set of all the partitions that are coarser than $X_{1}\left|X_{2}\right| \cdots \mid X_{k}$ and either exclude any subsystem of $Y_{1}\left|Y_{2}\right| \cdots \mid Y_{l}$ or include some but not all subsystems of $Y_{1}\left|Y_{2}\right| \cdots \mid Y_{l}$. We take the five-partite system $A B C D E$ for example,

$$
\begin{aligned}
\Xi(A|B| C D|E-A| B)= & \{C D|E, A| C D|E, B| C D|E, A| C D, A \mid E, \\
& B|E, A| C, A|D, B| C, B \mid D\}, \\
\Xi(A|B| C|D| E-A \mid C)= & \{B|D| E, B|D, B| E, D|E, A| B, A|D, A| E, \\
& B|C, C| D, C|E, A| B|D, A| B D, A B|D, A| B \mid E, \\
& A|B E, A B| E, A|D| E, A|D E, A D| E, B|C| D, \\
& B|C D, B C| D, B|C| E, B|C E, B C| E, B|D| E, \\
& B|D E, B D| E, C|D| E, C|D E, C D| E, A|B| D \mid E, \\
& B|C| D|E, A B| D E, B C|D E, A| B D E, A B D \mid E, \\
& B|C D E, B C D| E, A|B| D E, A B|D| E, A|B D| E, \\
& B C|D| E, B|C D| E, B|C| D E\} .
\end{aligned}
$$

For more clarity, we fix the following notations. Let $X_{1}\left|X_{2}\right| \cdots \mid X_{k}$ and $Y_{1}\left|Y_{2}\right| \cdots \mid Y_{l}$ be partitions of $A_{1} A_{2} \cdots A_{n}$ or subsystem of $A_{1} A_{2} \cdots A_{n}$. We denote by

$$
X_{1}\left|X_{2}\right| \cdots\left|X_{k} \succ^{a} Y_{1}\right| Y_{2}|\cdots| Y_{l}
$$

for the case of $(\mathrm{C} 1)$ and by,

$$
X_{1}\left|X_{2}\right| \cdots\left|X_{k} \succ^{b} Y_{1}\right| Y_{2}|\cdots| Y_{l}
$$


for the case of of $(\mathrm{C} 2)$, and in addition by

$$
X_{1}\left|X_{2}\right| \cdots\left|X_{k} \succ^{c} Y_{1}\right| Y_{2}|\cdots| Y_{l}
$$

for the case of (C3). For example, $A|B| C \succ^{a} A|B, A| B\left|C \succ^{b} A\right| B C, A\left|B C \succ^{c} A\right| B$, $A\left|B C \succ^{c} A\right| C$.

\subsection{Complete multipartite entanglement measure}

The counterpart to MQD for multipartite entanglement is the complete multipartite entanglement measure. A function $E^{(n)}: \mathcal{S}^{A_{1} A_{2} \cdots A_{n}} \rightarrow \mathbb{R}_{+}$is called an $n$-partite entanglement measure in literature [30] if it satisfies:

- (E1) $E^{(n)}(\rho)=0$ if $\rho$ is fully separable;

- (E2) $E^{(n)}$ cannot increase under $n$-partite LOCC.

When we take into consideration an $n$-partite measure of entanglement or other quantum correlation, we need discuss whether it is defined uniformly for any $k$-partite system at first, $k<n$. Let $Q^{(n)}$ be a multipartite measure (for entanglement or quantum discord, ect). If $Q^{(k)}$ is uniquely determined by $Q^{(n)}$ for any $2 \leq k<n$, then we call $Q^{(n)}$ a uniform measure. For example, MQD and GQD are uniquely defined for any $k$, thus they are uniform measures. The $n$-partite entanglement of formation [19] defined as $E_{f}^{(n)}(|\psi\rangle)=\frac{1}{2} \sum_{i=1}^{m} S\left(\rho^{A_{i}}\right)$ for pure state and via the convex-roof extension for mixed states (i.e., $E_{f}^{(n)}(\rho):=\min \sum_{i} p_{i} E_{f}^{(n)}\left(\left|\psi_{i}\right\rangle\right)$ for any mixed state $\rho$, where the minimum is taken over all pure-state decomposition $\left\{p_{i},\left|\psi_{i}\right\rangle\right\}$ of $\rho$ ), is a uniform multipartite entanglement measure. That is, a uniform measure is series of measures that have uniform expressions definitely. A uniform multipartite entanglement measure $E^{(n)}$ is called a unified multipartite entanglement measure if it also satisfies the following condition [19]:

- (E3) the unification condition, i.e., $E^{(n)}$ is consistent with $E^{(k)}$ for any $2 \leqslant k<n$.

The unification condition should be comprehended in the following sense [19]. Let $|\psi\rangle^{A_{1} A_{2} \cdots A_{n}}=|\psi\rangle^{A_{1} A_{2} \cdots A_{k}}|\psi\rangle^{A_{k+1} \cdots A_{n}}$, then

$$
\begin{aligned}
& E^{(n)}\left(|\psi\rangle^{A_{1} A_{2} \cdots A_{n}}\right) \\
= & E^{(k)}\left(|\psi\rangle^{A_{1} A_{2} \cdots A_{k}}\right)+E^{(n-k)}|\psi\rangle^{A_{k+1} \cdots A_{n}} .
\end{aligned}
$$

And

$$
E^{(n)}\left(\rho^{A_{1} A_{2} \cdots A_{n}}\right)=E^{(n)}\left(\rho^{\pi\left(A_{1} A_{2} \cdots A_{n}\right)}\right)
$$

for any $\rho^{A_{1} A_{2} \cdots A_{n}} \in \mathcal{S}^{A_{1} A_{2} \cdots A_{n}}$, where $\pi$ is a permutation of the subsystems. In addition,

$$
E^{(k)}\left(X_{1}\left|X_{2}\right| \cdots \mid X_{k}\right) \geqslant E^{(l)}\left(Y_{1}\left|Y_{2}\right| \cdots \mid Y_{l}\right)
$$

for any $\rho^{A_{1} A_{2} \cdots A_{n}} \in \mathcal{S}^{A_{1} A_{2} \cdots A_{n}}$ whenever $X_{1}\left|X_{2}\right| \cdots\left|X_{k} \succ^{a} \quad Y_{1}\right| Y_{2}|\cdots| Y_{l}$, where the vertical bar indicates the split across which the entanglement is measured. A uniform MEM $E^{(n)}$ is called a complete multipartite entanglement measure if it satisfies both (E3) above and the following [19]: 
- (E4) $E^{(n)}\left(X_{1}\left|X_{2}\right| \cdots \mid X_{k}\right) \geqslant E^{(k)}\left(Y_{1}\left|Y_{2}\right| \cdots \mid Y_{l}\right)$ holds for all $\rho \in \mathcal{S}^{A_{1} A_{2} \cdots A_{n}}$ whenever $X_{1}\left|X_{2}\right| \cdots\left|X_{k} \succ^{b} Y_{1}\right| Y_{2}|\cdots| Y_{l}$.

It is easy to see that $E_{f}^{(n)}$ is a complete multipartite entanglement measure [19].

\subsection{Monogamy relation}

It is known that classical correlations can be freely shared among many parties, i.e., A party A can have maximal classical correlations with two parties B and $\mathrm{C}$ simultaneously. But this is no longer the case if quantum entanglement or other nonlocal correlations are concerned [16]. The impossibility of sharing those types of nonclassical correlations unconditionally across many parties are known as monogamy constraints. For a given bipartite measure of nonlocal correlation $Q, Q$ is said to be monogamous (we take the tripartite case for example) if $[14,16]$

$$
Q(A \mid B C) \geq Q(A B)+Q(A C) .
$$

However, Eq. (12) is not valid for many entanglement measures [14, 16] and quantum discord [24] but some power function of $Q$ admits the monogamy relation, i.e., $Q^{\alpha}(A \mid B C) \geq Q^{\alpha}(A B)+Q^{\alpha}(A C)$ for some $\alpha>0$. In Ref. [17], we address this issue by proposing an improved definition of monogamy (without inequalities) for entanglement measure: A measure of entanglement $E$ is monogamous if for any $\rho \in \mathcal{S}^{A B C}$ that satisfies the disentangling condition, i.e.,

$$
E\left(\rho^{A \mid B C}\right)=E\left(\rho^{A B}\right),
$$

we have that $E\left(\rho^{A C}\right)=0$. With respect to this definition, a continuous measure $E$ is monogamous according to this definition if and only if there exists $0<\alpha<\infty$ such that

$$
E^{\alpha}\left(\rho^{A \mid B C}\right) \geq E^{\alpha}\left(\rho^{A B}\right)+E^{\alpha}\left(\rho^{A C}\right),
$$

for all $\rho$ acting on the state space $\mathcal{H}^{A B C}$ with fixed $\operatorname{dim} \mathcal{H}^{A B C}=d<\infty$ (see Theorem 1 in Ref. [17]). With this improved definition of monogamy, we proved that almost all the bipartite entanglement measures so far are monogamous [17, 18]. Notice that, for these bipartite measures, only the relation between $A \mid B C, A B$ and $A C$ are revealed, the global correlation in $\mathrm{ABC}$ and the correlation contained in part $B C$ is missed [19]. That is, the monogamy relation in such a sense is not "complete". Recently, we established a complete monogamy relation for entanglement in Ref. [19]. For a unified tripartite entanglement measure $E^{(3)}$, it is said to be completely monogamous if is satisfies the complete disentangling condition [19], i.e., for any $\rho \in \mathcal{S}^{A B C}$ that satisfies

$$
E^{(3)}\left(\rho^{A B C}\right)=E^{(2)}\left(\rho^{A B}\right)
$$

we have that $E^{(2)}\left(\rho^{A C}\right)=E^{(2)}\left(\rho^{B C}\right)=0$. If $E^{(3)}$ is a continuous unified tripartite entanglement measure. Then, $E^{(3)}$ is completely monogamous if and only if there exists $0<\alpha<\infty$ such that $[19]$

$$
E^{\alpha}\left(\rho^{A B C}\right) \geq E^{\alpha}\left(\rho^{A B}\right)+E^{\alpha}\left(\rho^{A C}\right)+E^{\alpha}\left(\rho^{B C}\right),
$$


for all $\rho^{A B C} \in \mathcal{S}^{A B C}$ with fixed $\operatorname{dim} \mathcal{H}^{A B C}=d<\infty$, here we omitted the superscript $(2,3)$ of $E^{(2,3)}$ for brevity.

Let $E^{(3)}$ be a complete MEM. $E^{(3)}$ is defined to be tightly complete monogamous if it satisfies the tight disentangling conditon, i.e., for any state $\rho^{A B C} \in \mathcal{S}^{A B C}$ that satisfying [19]

$$
E^{(3)}\left(\rho^{A B C}\right)=E^{(2)}\left(\rho^{A \mid B C}\right)
$$

we have $E^{(2)}\left(\rho^{B C}\right)=0$, which is equivalent to

$$
E^{\alpha}\left(\rho^{A B C}\right) \geqslant E^{\alpha}\left(\rho^{A \mid B C}\right)+E^{\alpha}\left(\rho^{B C}\right)
$$

for some $\alpha>0$, here we omitted the superscript ${ }^{(2,3)}$ of $E^{(2,3)}$ for brevity. For the general case of $E^{(m)}$, one can similarly process with the same spirit.

\section{Framework of complete monogamy relation for quantum discord}

All the previous discussions on the monogamy for quantum discord in literatures are based on the bipartite quantum discord as in Eq. (12). The complete monogamy relation in Eq. (15) displays the distribution of the correlation throughly. We thus adopt this scenario to describe the monogamy of MQD and GQD, namely, we consider the complete monogamy relation as Eqs. (15) and (17) with MQD/GQD replacing entanglement measure $E$.

According to the complete multipartite entanglement measure, for a uniform measure of quantum correlation $Q^{(m)}$, we expect it satisfies the following conditions when

we discuss the complete monogamy relation [we take the tripartite case for example, the $m$-partite $(m \geq 4)$ case can be argued similarly]:

$$
\begin{aligned}
& \text { (U1) }: Q^{(3)}\left(\rho^{A B} \otimes \rho^{C}\right)=Q^{(2)}\left(\rho^{A B}\right), \quad \forall \rho^{A B} \otimes \rho^{C} \in \mathcal{S}^{A B C} . \\
& \text { (U2) }: Q^{(3)}\left(\rho^{A B C}\right)=Q^{(3)}\left(\rho^{\pi(A B C)}\right), \quad \forall \rho^{A B C} \in \mathcal{S}^{A B C} . \\
& \text { (U3) }: Q^{(3)}(A B C) \geq Q^{(2)}(X Y), \quad \forall \rho^{A B C} \in \mathcal{S}^{A B C} . \\
& \text { (U4) }: Q^{(3)}(A B C) \geq Q^{(2)}(X \mid Y Z), \quad \forall \rho^{A B C} \in \mathcal{S}^{A B C} .
\end{aligned}
$$

We now begin to check whether MQD and GQD are unified. It is proved in Ref. [11] that $D_{A ; B ; C}=D_{X ; Y}$ for any $\rho=\rho^{X Y} \otimes \rho^{Z} \in \mathcal{S}^{A B C},\{X, Y, Z\}=\{A, B, C\}$. Namely, MQD satisfies (U1). Going further, for any bipartite state $\rho \in \mathcal{S}^{A B}$, for any given decomposition $\rho=\sum_{i} p_{i} \rho_{i}^{A B}$, it can be extended by adding an auxiliary system $C$ that does not correlate with $A B$ as

$$
\rho=\sum_{i} p_{i}|i\rangle\left\langle\left. i\right|^{C} \otimes \rho_{i}^{A B} .\right.
$$

That is, $D_{C ; A B}=0$. One can easily show that

$$
D_{C ; A ; B}=D_{A ; B}
$$


for such a state. We can also show that (see in Proposition 1 and Proposition 2 in the next Section)

$$
D_{A ; C ; B} \geq D_{A ; B}
$$

with the equality holds if and only if $D_{A ; C}=0$ for such a state. Eqs. $(23,24)$ imply that: (i) when an auxiliary particle classically correlated with the state is added and it does not disturb the measurement ordering $A \rightarrow B$, then the MQD equals to the pre-state; (ii) when the auxiliary particle disturb the previous measurement ordering $A \rightarrow B$, then the multipartite discord does not decrease. That is MQD obeys more than (U1). But MQD violates (U2) obviously. The GQD satisfies (U1) since $D_{A: B: C}\left(\rho^{A B} \otimes \rho^{C}\right)=D_{A: B}\left(\rho^{A B}\right)$ and (U2) is clear since it is symmetric. However, for these measurement-induced correlations, whether they obey conditions (U3) and (U4), is not straightforward.

We are now ready to discuss the complete monogamy of these two generalizations of quantum discord. With the same principle as the unified/complete multiparty entanglement measure and the complete monogamy of entanglement introduced in the previous section, we can now give the definition of complete multiparty quantum discord and complete monogamy for MQD and GQD.

Definition 1. Let $D_{A_{1}\left|A_{2}\right| \cdots \mid A_{n}}$ be $D_{A_{1} ; A_{2} ; \cdots ; A_{n}}$ or $D_{A_{1}: A_{2}: \cdots: A_{n}}$ defined on $\mathcal{S}^{A_{1} A_{2} \cdots A_{n}}$. With the notations aforementioned, $D_{A_{1}\left|A_{2}\right| \cdots \mid A_{n}}$ is said to be complete if it is monotonic under coarsening of subsystem(s), i.e., for any $\rho \in \mathcal{S}^{A_{1} A_{2} \cdots A_{n}}$, we have

$$
D_{X_{1}\left|X_{2}\right| \cdots \mid X_{k}} \geq D_{Y_{1}\left|Y_{2}\right| \cdots \mid Y_{l}}
$$

holds for any partitions $X_{1}\left|X_{2}\right| \cdots \mid X_{k}$ and $Y_{1}\left|Y_{2}\right| \cdots \mid Y_{l}$ of $A_{1} A_{2} \cdots A_{m}$ or subsystem of $A_{1} A_{2} \cdots A_{m}$ with $X_{1}\left|X_{2}\right| \cdots\left|X_{k} \succ Y_{1}\right| Y_{2}|\cdots| Y_{l}$ whenever $D_{A_{1}\left|A_{2}\right| \cdots \mid A_{n}}=D_{A_{1} ; A_{2} ; \cdots ; A_{n}}$ and with $X_{1}\left|X_{2}\right| \cdots\left|X_{k} \succ^{a, b} Y_{1}\right| Y_{2}|\cdots| Y_{l}$ whenever $D_{A_{1}\left|A_{2}\right| \cdots \mid A_{n}}=D_{A_{1}: A_{2}: \cdots: A_{n}}$.

Definition 2. Let $D_{A_{1}\left|A_{2}\right| \cdots \mid A_{n}}$ be $D_{A_{1} ; A_{2} ; \cdots ; A_{n}}$ or $D_{A_{1}: A_{2}: \cdots: A_{n}}$ defined on $\mathcal{S}^{A_{1} A_{2} \cdots A_{n}}$. With the notations aforementioned, $D_{A_{1}\left|A_{2}\right| \cdots \mid A_{n}}$ is said to be completely monogamous if it is complete and satisfies the dis-correlated condition, i.e., for any state $\rho \in \mathcal{S}^{A_{1} A_{2} \cdots A_{n}}$ that satisfies

$$
D_{X_{1}\left|X_{2}\right| \cdots \mid X_{k}}=D_{Y_{1}\left|Y_{2}\right| \cdots \mid Y_{l}}
$$

we have that

$$
D_{\Gamma}=0
$$

holds for all $\Gamma \in \Xi\left(X_{1}\left|X_{2}\right| \cdots\left|X_{k}-Y_{1}\right| Y_{2}|\cdots| Y_{l}\right)$, where $X_{1}\left|X_{2}\right| \cdots \mid X_{k}$ and $Y_{1}\left|Y_{2}\right| \cdots \mid Y_{l}$ are arbitrarily given partitions of $A_{1} A_{2} \cdots A_{m}$ or subsystem of $A_{1} A_{2} \cdots A_{m}$, and where $X_{1}\left|X_{2}\right| \cdots\left|X_{k} \succ Y_{1}\right| Y_{2}|\cdots| Y_{l}$ whenever $D_{A_{1}\left|A_{2}\right| \cdots \mid A_{n}}=D_{A_{1} ; A_{2} ; \cdots ; A_{n}}, X_{1}\left|X_{2}\right| \cdots \mid X_{k} \succ^{a, b}$ $Y_{1}\left|Y_{2}\right| \cdots \mid Y_{l}$ whenever $D_{A_{1}\left|A_{2}\right| \cdots \mid A_{n}}=D_{A_{1}: A_{2}: \cdots: A_{n}}$.

That is, MQD/GQD is complete if and only if it satisfies the conditions (U3) and (U4). Namely, for MQD and GQD, we do not distinguish conditions (U3) and (U4) which are corresponding to the unified measure and complete measure for entanglement respectively, and call it complete for both of them uniformly. In other 
words, MQD/GQD is a complete multiparty quantum discord if Eq. (25) hold true. Condition (26) is the counterpart to the complete disentangling condition together with the tight disentangling condition for multipartite entanglement. It is not necessary to distinguish these two disentangling conditions for MQD and GQD. In what follows, both the complete disentangling condition and the tight disentangling condition for multipartite entanglement are called complete disentangling condition for simplicity.

We illustrate Definitions 1 and 2 with the four partite case. $D_{A ; B ; C ; D}$ is complete if: For any state $\rho \in \mathcal{S}^{A B C D}, D_{A ; B ; C ; D} \geq D_{X ; Y ; Z} \geq D_{M ; N}$ for any $\{M, N\} \subseteq$ $\{X, Y, Z\} \subseteq\{A, B, C, D\}$ with ordering $A \rightarrow B \rightarrow C \rightarrow D$ for both $M, N$ and $X, Y, Z$. In addition, $D_{A ; B ; C ; D} \geq D_{A ; B C ; D} \geq D_{A ; B C} \geq D_{A ; B}, D_{A ; B ; C ; D} \geq D_{A ; B C D} \geq$ $D_{A ; B C} \geq D_{A ; C}, D_{A ; B ; C ; D} \geq D_{A B ; C D} \geq D_{A B ; C}, D_{A B ; C D} \geq D_{A B ; D}, D_{A ; B ; C ; D} \geq D_{A B C ; D}$, $D_{A ; B ; C ; D} \geq D_{A B ; C ; D} \geq D_{A B ; C}, D_{A B ; C ; D} \geq D_{A B ; D}, D_{A ; B ; C ; D} \geq D_{A ; B ; C D} \geq D_{A B ; C D}$, $D_{A ; B ; C D} \geq D_{A ; B ; C}, D_{A ; B ; C D} \geq D_{A ; B ; D} . D_{A ; B ; C ; D}$ is completely monogamous if: For any state $\rho \in \mathcal{S}^{A B C D}$ that satisfies $D_{A ; B ; C ; D}=D_{A ; B ; C}$, we have that $D_{A ; B ; D}=D_{A ; C ; D}=$ $D_{B ; C ; D}=D_{A ; D}=D_{B ; D}=D_{C ; D}=0$. For any state $\rho \in \mathcal{S}^{A B C D}$ that satisfies $D_{A ; B ; C ; D}=D_{A ; B}$, we have that $D_{C ; D}=D_{A ; C ; D}=D_{B ; C ; D}=D_{A ; C}=D_{A ; D}=D_{B ; C}=$ $D_{B ; D}=0$. Similarly, for any state $\rho \in \mathcal{S}^{A B C D}$ that satisfies $D_{A ; B ; C ; D}=D_{A ; C}$, we have that $D_{A ; B}=D_{B ; D}=D_{A ; B ; D}=D_{B ; C ; D}=D_{A ; D}=D_{B ; C}=D_{C ; D}=0$. In addition, for any state $\rho \in \mathcal{S}^{A B C D}$ that satisfies $D_{A ; B ; C ; D}=D_{A B ; C}$, we have that $D_{A ; B}=D_{A B ; D}=D_{C ; D}=0$. The other cases can be argued similarly. $D_{A: B: C: D}$ is complete if: For any state $\rho \in \mathcal{S}^{A B C D}, D_{A: B: C: D} \geq D_{X: Y: Z} \geq D_{M: N}$ for any $\{M, N\} \subseteq\{X, Y, Z\} \subseteq\{A, B, C, D\}$. In addition, $D_{A: B: C: D} \geq D_{A: B C: D} \geq D_{A: B C}$, $D_{A: B: C: D} \geq D_{A: B C D}, D_{A: B: C: D} \geq D_{A B: C D}, D_{A: B: C: D} \geq D_{A B C: D}, D_{A: B: C: D} \geq D_{A B: C: D} \geq$ $D_{A B: C}, D_{A B: C: D} \geq D_{A B: D}, D_{A: B: C: D} \geq D_{A: B: C D} \geq D_{A B: C D}$. $D_{A: B: C: D}$ is completely monogamous if: For any state $\rho \in \mathcal{S}^{A B C D}$ that satisfies $D_{A: B: C: D}=D_{A: B: C}$, we have that $D_{A: B: D}=D_{A: C: D}=D_{B: C: D}=D_{A: D}=D_{B: D}=D_{C: D}=0$. For any state $\rho \in \mathcal{S}^{A B C D}$ that satisfies $D_{A: B: C: D}=D_{A: B}$, we have that $D_{C: D}=D_{A: C: D}=D_{B: C: D}=D_{A: C}=D_{A: D}=$ $D_{B: C}=D_{B: D}=0$. Similarly, for any state $\rho \in \mathcal{S}^{A B C D}$ that satisfies $D_{A: B: C: D}=D_{A: C}$ , we have that $D_{A: B}=D_{B: D}=D_{A: B: D}=D_{B: C: D}=D_{A: D}=D_{B: C}=D_{C: D}=0$. In addition, for any state $\rho \in \mathcal{S}^{A B C D}$ that satisfies $D_{A: B: C: D}=D_{A B: C}$, we have that $D_{A: B}=D_{A B: D}=D_{C: D}=0$. The other cases can be processed analogously.

Remark 1. We call it complete monogamy here for MQD and GQD since both MQD and GQD are multipartite measures, and this is consistent with the complete and tightly complete monogamy for multipartite entanglement measure. The previous research in this field is based on the bipartite measure of quantum discord [24, 25, 26, 27, 28] and it is called monogamy but not complete monogamy. This is analogous to that of bipartite entanglement measure in which it is called monogamy but not complete monogamy. That is, for both quantum discord and entanglement, 'complete' refers to the uniform multipartite measure and the one without 'complete' refers to the bipartite measure.

Remark 2. Comparing with the definition of the complete monogamy for entanglement, the difference is that, for entanglement we only require the condition (26), i.e., the complete disentangling condition for entanglement (see Eqs. (13) and (15)). 
Condition (25) is true naturally for any unified multipartite entanglement measure $E^{(m)}$ since it is decreasing under tracing out any subsystem. But this fact is not obvious for other quantum correlation. So the monogamy of quantum discord need this assumption necessarily.

Remark 3. For MQD, Eq. (25) in Definition 1 and Eq. (26) in Definition 2 are presented under the fixed ording $A_{1} \rightarrow A_{2} \rightarrow \ldots \rightarrow A_{n-1} \rightarrow A_{n}$ since MQD strictly depends on the ordering of the measurements on subsystems. But it is superfluous for the globle quantum discord since it admits (U2).

Remark 4. In Definition 1 and Definition 2, MQD and GQD are different: The coarser relation of class (C3) is meaningless for GQD since in such a case the corresponding measurements are not compatible as we argued in Subsection 2.2. It has been shown that there exists three-qubit state $\rho \in \mathcal{S}^{A B C}$ such that $D_{A: B C}<D_{A: B}[23]$. However, althouhgh $D_{A: B C}<D_{A: B}$ for some state, it seems true that $D_{A: B: C}>D_{M: N}$ for any tripartite state (also see the assumption in Proposition 9), $\{M, N\} \subseteq\{A, B, C\}$. That is, the monotonicity should holds whenever the measurement on both sides are the same one. But for the case of MQD, such a case can not occur since the last subsystem is not measured, so the remaining measurements are always compatible. This is why we require three classes of coarsening relations when we camparing $D_{X_{1} ; X_{2} ; \cdots ; X_{k}}$ and $D_{Y_{1} ; Y_{2} ; \cdots ; Y_{l}}$, and only require coarsening classes of $(\mathrm{C} 1)$ and $(\mathrm{C} 2)$ for that of GQD in these definitions.

\section{Monogamy of the multipartite quantum discord}

For convenience, we fix some notations. For any local measurement $\Pi^{A_{i_{1}} \cdots A_{i_{k}}}$ acting on the reduced state $\rho^{A_{i_{1}} \cdots A_{i_{k}} A_{i_{k+1}}}$ of $\rho^{A_{1} A_{2} \cdots A_{n}} \in \mathcal{S}^{A_{1} A_{2} \cdots A_{n}}$, the conditional mutual information changes by an amount

$$
d_{A_{i_{1}} \ldots A_{i_{k}} ; A_{i_{k+1}}}=S_{A_{i_{k+1}} \mid \Pi^{A_{i_{1}} \ldots A_{i_{k}}}}-S_{A_{i_{k+1}} \mid A_{i_{1}} \ldots A_{i_{k}}}
$$

where $1 \leq i_{1}<i_{2}<\cdots i_{k}<i_{k+1} \leq n$.

\subsection{The tripartite case}

Different from multipartite entanglement, it is unknown whether the correlation decreases under coarsening the subsystems.

Proposition 1. For any state $\rho$ in $\mathcal{S}^{A B C}$, the following coarsening relations hold:

- $D_{A ; B ; C} \geq D_{A ; B}+D_{A B ; C}, D_{A ; B ; C} \geq D_{A ; C}$.

- $D_{A ; B ; C} \geq D_{B ; C}$ provided that $d_{A B ; C} \geq d_{B ; C}$.

- $D_{A ; B ; C} \geq D_{A ; B C} \geq D_{A ; B}$.

- $D_{A ; B C} \geq D_{A ; C}$ provided that $d_{A B ; C} \geq d_{A ; C}$.

That is, if $d_{A B ; C} \geq d_{B ; C}$ and $d_{A B ; C} \geq d_{A ; C}$, then $D_{A ; B ; C}$ is a complete multiparty quantum discord. 
Proof. We assume that $D_{A ; B}\left(\rho^{A B}\right)=S\left(\rho^{A B}\right)-S\left(\rho^{A}\right)-S\left(\rho^{A B}\right)+S\left(\rho^{A}\right)$ for some $\Pi^{A}\left(\rho^{A B}\right)=\rho^{A B}$, and that $D_{A ; B ; C}\left(\rho^{A B C}\right)=S\left(\rho^{\prime \prime A B}\right)-S\left(\rho^{\prime \prime A}\right)+S\left(\rho^{\prime \prime \prime A B C}\right)-S\left(\rho^{\prime \prime \prime} A B\right)-$ $S\left(\rho^{A B C}\right)+S\left(\rho^{A}\right)$ for some $\Pi^{\prime A B}$ with $\Pi^{\prime A}\left(\rho^{A B C}\right)=\rho^{\prime \prime A B C}$ and $\Pi^{\prime A B}\left(\rho^{A B C}\right)=\rho^{\prime \prime \prime A B C}$. (Hereafter, in this subsection, we denote by $\rho^{\prime}$ the state after the optimal local measurement on one particle of the bipartite state, by $\rho^{\prime \prime}$ the state after the optimal local measurement on one particle of the tripartite state, and by $\rho^{\prime \prime \prime}$ the state after the optimal local measurement on two particles of the bipartite state.) For simplicity, we denote $S\left(\rho^{* X}\right)$ by $S_{X}^{*}$ hereafter (e.g., we denote $S\left(\rho^{\prime \prime A B}\right)$ by $S_{A B}^{\prime \prime}$ for brevity). It is straightforward that, for any given $\rho \in \mathcal{S}^{A B C}$, we have

$$
\begin{aligned}
& D_{A ; B ; C}-D_{A ; B} \\
= & \min _{\Pi^{A B}}\left(-S_{B C \mid A}+S_{B \mid \Pi^{A}}+S_{C \mid \Pi^{A B}}\right)-\min _{\Pi^{A}}\left(S_{B \mid \Pi^{A}}-S_{B \mid A}\right) \\
= & \left(S_{A B}^{\prime \prime}-S_{A}^{\prime \prime \prime}+S_{A B C}^{\prime \prime \prime}-S_{A B}^{\prime \prime \prime}-S_{A B C}+S_{A}\right)-\left(S_{A B}^{\prime \prime}-S_{A}^{\prime \prime}-S_{A B}+S_{A}\right) \\
& +\left(S_{A B}^{\prime \prime}-S_{A}^{\prime \prime}-S_{A B}+S_{A}\right)-\left(S_{A B}^{\prime}-S_{A}^{\prime}-S_{A B}+S_{A}\right) \\
\geq & \left(S_{A B}^{\prime \prime}-S_{A}^{\prime \prime}+S_{A B C}^{\prime \prime \prime}-S_{A B}^{\prime \prime \prime}-S_{A B C}+S_{A}\right)-\left(S_{A B}^{\prime \prime}-S_{A}^{\prime \prime \prime}-S_{A B}+S_{A}\right) \\
= & S_{A B C}^{\prime \prime \prime}-S_{A B}^{\prime \prime \prime}-S_{A B C}+S_{A B} \\
\geq & D_{A B ; C} .
\end{aligned}
$$

Note that, even thought the optimal local measurement does not exist, we have that, for any $\epsilon>0$, there exist $\Pi^{A}$ and $\Pi^{\prime A B}$ such that $D_{A ; B ; C} \geq S\left(\rho^{\prime \prime A B}\right)-S\left(\rho^{\prime \prime A}\right)+S\left(\rho^{\prime \prime \prime A B C}\right)-S\left(\rho^{\prime \prime \prime A B}\right)-S\left(\rho^{A B C}\right)+S\left(\rho^{A}\right)-$ $\epsilon$ and $S\left(\rho^{\prime \prime A B}\right)-S\left(\rho^{\prime \prime A}\right)-S\left(\rho^{A B}\right)+S\left(\rho^{A}\right) \geq D_{A ; B}$. It turns out that $D_{A ; B ; C}-D_{A ; B} \geq\left[S\left(\rho^{\prime \prime A B}\right)-S\left(\rho^{\prime \prime A}\right)+S\left(\rho^{\prime \prime \prime A B C}\right)-S\left(\rho^{\prime \prime \prime A B}\right)-S\left(\rho^{A B C}\right)+S\left(\rho^{A}\right)\right]-$ $\left[S\left(\rho^{\prime \prime A B}\right)-S\left(\rho^{\prime \prime A}\right)-S\left(\rho^{A B}\right)+S\left(\rho^{A}\right)\right]-\epsilon \geq 0$ is equivalent to $\left[S\left(\rho^{\prime \prime A B}\right)-S\left(\rho^{\prime \prime A}\right)+\right.$ $\left.S\left(\rho^{\prime \prime \prime A B C}\right)-S\left(\rho^{\prime \prime \prime A B}\right)-S\left(\rho^{A B C}\right)+S\left(\rho^{A}\right)\right]-\left[S\left(\rho^{\prime \prime A B}\right)-S\left(\rho^{\prime \prime A}\right)-S\left(\rho^{A B}\right)+S\left(\rho^{A}\right)\right] \geq 0$ since $\epsilon$ can be arbitrarily small. So we can assume with no loss of generality that the optimal local measurement always exists hereafter. Similarly,

$$
\begin{aligned}
& D_{A ; B ; C}-D_{A ; C} \\
\geq & \left(S_{A B}^{\prime \prime}-S_{A}^{\prime \prime}+S_{A B C}^{\prime \prime \prime}-S_{A B}^{\prime \prime \prime}-S_{A B C}+S_{A}\right)-\left(S_{A C}^{\prime \prime}-S_{A}^{\prime \prime}-S_{A C}+S_{A}\right) \\
= & \left(S_{A B C}^{\prime \prime \prime}-S_{A B}^{\prime \prime \prime}-S_{A B C}+S_{A B}\right)+\left(S_{A B C}^{\prime \prime}-S_{A C}^{\prime \prime}-S_{A B C}+S_{A C}\right) \\
& -\left(S_{A B C}^{\prime \prime}-S_{A B}^{\prime \prime}-S_{A B C}+S_{A B}\right) \\
\geq & \left(S_{A B C}^{\prime \prime \prime}-S_{A B}^{\prime \prime \prime}-S_{A B C}+S_{A B}\right)-\left(S_{A B C}^{\prime \prime}-S_{A B}^{\prime \prime}-S_{A B C}+S_{A B}\right) \\
= & S_{A B C}^{\prime \prime \prime}-S_{A B}^{\prime \prime \prime}-S_{A B C}^{\prime \prime}+S_{A B}^{\prime \prime} \\
\geq & 0 .
\end{aligned}
$$

Here, the second inequality holds since the mutual information always decreases under local operation [31] (which is equivalent to $S_{X Y}^{\prime \prime}-S_{X}^{\prime \prime}-S_{X Y}+S_{X} \geq 0$ ).

Observe that

$$
\begin{aligned}
& D_{A ; B ; C}-D_{B ; C} \\
\geq & \left(S_{A B}^{\prime \prime}-S_{A}^{\prime \prime}+S_{A B C}^{\prime \prime \prime}-S_{A B}^{\prime \prime \prime}-S_{A B C}+S_{A}\right)-\left(S_{B C}^{\prime \prime \prime}-S_{B}^{\prime \prime \prime}-S_{B C}^{\prime \prime}+S_{B}^{\prime \prime}\right)
\end{aligned}
$$


Monogamy of quantum discord

$$
\begin{aligned}
= & \left(S_{A B}^{\prime \prime \prime}-S_{A}^{\prime \prime}+S_{A B C}^{\prime \prime \prime}-S_{A B}^{\prime \prime \prime}-S_{A B C}+S_{A}\right)-\left(S_{B C}^{\prime \prime \prime}-S_{B}^{\prime \prime \prime}-S_{B C}+S_{B}\right) \\
= & \left(S_{A B C}^{\prime \prime \prime}-S_{A B}^{\prime \prime \prime}-S_{A B C}+S_{A B}\right)+\left(S_{A B}^{\prime \prime}-S_{A}^{\prime \prime \prime}-S_{A B}+S_{A}\right) \\
& -\left(S_{B C}^{\prime \prime \prime \prime}-S_{B}^{\prime \prime \prime}-S_{B C}+S_{B}\right),
\end{aligned}
$$

thus $D_{A ; B ; C} \geq D_{B ; C}$ since $d_{A B ; C} \geq d_{B ; C}$ by assumption. The following calculation are clear. $D_{A ; B ; C}-D_{A ; B C} \geq\left(S_{A B}^{\prime \prime}-S_{A}^{\prime \prime}+S_{A B C}^{\prime \prime \prime}-S_{A B}^{\prime \prime \prime}-S_{A B C}+S_{A}\right)-\left(S_{A B C}^{\prime \prime}-S_{A}^{\prime \prime}-\right.$ $\left.S_{A B C}+S_{A}\right)=\left(S_{A B}^{\prime \prime}-S_{A}^{\prime \prime}+S_{A B C}^{\prime \prime \prime}-S_{A B}^{\prime \prime \prime}-S_{A B C}+S_{A}\right)-\left(S_{A B C}^{\prime \prime}-S_{A}^{\prime \prime}-S_{A B C}+S_{A}\right)=$ $\left(S_{A B C}^{\prime \prime \prime}-S_{A B}^{\prime \prime \prime}-S_{A B C}^{\prime \prime}+S_{A B}^{\prime \prime}\right) \geq 0$ implies $D_{A ; B ; C} \geq D_{A ; B C} . \quad D_{A ; B C}-D_{A ; B} \geq$ $\left(S_{A B C}^{\prime \prime}-S_{A}^{\prime \prime}-S_{A B C}+S_{A}\right)-\left(S_{A B}^{\prime \prime}-S_{A}^{\prime \prime}-S_{A B}+S_{A}\right)=\left(S_{A B C}^{\prime \prime}-S_{A B}^{\prime \prime}-S_{A B C}+S_{A B}\right) \geq 0$, that is, $D_{A ; B C} \geq D_{A ; B} . D_{A ; B C}-D_{A ; C} \geq\left(S_{A B C}^{\prime \prime}-S_{A}^{\prime \prime}-S_{A B C}+S_{A}\right)-\left(S_{A B}^{\prime \prime}-S_{A}^{\prime \prime}-S_{A C}+S_{A}\right) \geq 0$ since $d_{A B ; C} \geq d_{A ; C}$ by assumption. This completes the proof.

Proposition 2. $D_{A ; B ; C}$ is completely monogamous if it is complete.

Proof. We use the notations in the proof of Proposition 1.

Case 1: $D_{A ; B ; C}=D_{A ; B}$. If $D_{A ; B ; C}\left(\rho^{A B C}\right)=D_{A ; B}\left(\rho^{A B}\right)$, we get $S\left(\rho^{\prime \prime \prime A B C}\right)-$ $S\left(\rho^{\prime \prime \prime} A B\right)-S\left(\rho^{A B C}\right)+S\left(\rho^{A B}\right)=0$ for some von Neumann measurement $\Pi^{A B}\left(\rho^{A B C}\right)=$ $\rho^{\prime \prime \prime} A B C$, which implies that $\rho^{A B C}=\sum_{k, j} p_{k, j}|k\rangle\left\langle\left. k\right|^{A} \otimes \mid j\right\rangle\left\langle\left. j\right|^{B} \otimes \rho_{k, j}^{C}\right.$. It is clear that $D_{A ; C}=D_{B ; C}=0$ for such a state.

Case 2: $D_{A ; B ; C}=D_{B ; C}$. According to Eq. (29), for any state $\rho \in \mathcal{S}^{A B C}$, if $D_{A ; B ; C}=D_{B ; C}$, then $S_{A B C}^{\prime \prime}-S_{A}^{\prime \prime}-S_{A B C}+S_{A}=I_{B C \mid A}-I_{B C \mid A}^{\prime \prime}=0$. Therefore $\rho=\sum_{k} p_{k}|k\rangle\left\langle\left. k\right|^{A} \otimes \rho_{k}^{B C}\right.$ for some basis $\left\{|k\rangle^{A}\right\}$ of $\mathcal{H}^{A}$, and thus $D_{A ; B}=D_{A ; C}=0$.

Case 3: If $D_{A ; B ; C}=D_{A ; C}$, one can easily check that $\rho=\sum_{k, j} p_{k, j}|k\rangle\left\langle\left. k\right|^{A} \otimes \mid j\right\rangle\left\langle\left. j\right|^{B} \otimes\right.$ $\rho_{k, j}^{C}$ for some basis $\left\{|k\rangle^{A}|j\rangle^{B}\right\}$ in $\mathcal{H}^{A B}$. It follows that $D_{A ; B}=D_{B ; C}=0$.

The other two cases $D_{A ; B ; C}=D_{A ; B C}$ and $D_{A ; B ; C}=D_{A ; B C}$ can be checked analogously.

Using the same notations as in the proof of Proposition 1, it is clear that, for any tripartite state $\rho \in \mathcal{S}^{A B C}$,

$$
\begin{aligned}
& D_{A ; B ; C}-D_{A ; B}-D_{A ; C} \\
\geq & \left(S_{A B C}^{\prime \prime \prime}-S_{A B}^{\prime \prime \prime}-S_{A B C}+S_{A B}\right)-\left(S^{\prime \prime \prime A C}-S^{\prime \prime \prime A}-S_{A C}+S_{A}\right) \\
\geq & 0
\end{aligned}
$$

whenever $d_{A B ; C} \geq d_{A ; C}$. That is

Proposition 3. For any state $\rho \in \mathcal{S}^{A B C}$ that satisfies $d_{A B ; C} \geq d_{A ; C}$, we have

$$
D_{A ; B ; C} \geq D_{A ; B}+D_{A ; C} .
$$

\subsection{The four-partite case}

With the increasing of particles involved, the hierarchy of coarsening relations become more complicated.

Proposition 4. For any state $\rho$ in $\mathcal{S}^{A B C D}$, the following coarsening relations hold:

- $D_{A ; B ; C ; D} \geq D_{A ; B ; C}+D_{A B C ; D}$. 
- $D_{A ; B ; C ; D} \geq D_{A ; B ; D}$.

- $D_{A ; B ; C ; D} \geq D_{A ; B}, D_{A ; B ; C ; D} \geq D_{A ; C}, D_{A ; B ; C ; D} \geq D_{A ; D}$.

- $D_{A ; B ; C ; D} \geq D_{A ; C ; D}$ provided that $d_{A B C ; D} \geq d_{A C ; D}$.

- $D_{A ; B ; C ; D} \geq D_{A ; C ; D}+D_{A ; B}$ provided that $d_{A B C ; D} \geq d_{A C ; D}$ and $d_{A B ; C} \geq d_{A ; C}$.

- $D_{A ; B ; C ; D} \geq D_{B ; C ; D}+D_{A ; B}$ provided that $d_{A B C ; D} \geq d_{B C ; D}$ and $d_{A B ; C} \geq d_{B ; C}$.

- $D_{A ; B ; C ; D} \geq D_{A ; B ; D}+D_{A B ; C}$ provided that $d_{A B C ; D} \geq d_{A B ; D}$.

- $D_{A ; B ; C ; D} \geq D_{B ; C}$ provided that $d_{A B ; C} \geq d_{B ; C}$ or $d_{A B C ; D} \geq d_{B ; C}$.

- $D_{A ; B ; C ; D} \geq D_{B ; D}$ provided that $d_{A B C ; D} \geq d_{B ; D}$.

- $D_{A ; B ; C ; D} \geq D_{C ; D}$ provided that $d_{A B C ; D} \geq d_{A ; D}$.

- $D_{A ; B ; C ; D} \geq D_{A B ; C D}+D_{A ; B}$.

- $D_{A ; B ; C ; D} \geq D_{A ; B C D}$.

- $D_{A ; B ; C ; D} \geq D_{A ; B C ; D}$.

- $D_{A ; B ; C ; D} \geq D_{A B ; C ; D}+D_{A ; B}$.

- $D_{A ; B ; C ; D} \geq D_{A ; B ; C D}$.

That is, $D_{A ; B ; C ; D}$ is a complete multiparty quantum discord if $d_{A B C ; D} \geq d_{A B ; D}$, $d_{A B C ; D} \geq d_{A C ; D}, d_{A B C ; D} \geq d_{B C ; D}, d_{A B ; C} \geq d_{A ; C}$, and $d_{A B ; C} \geq d_{B ; C}$.

Proof. For any $\rho \in \mathcal{S}^{A B C D}$, we assume that $D_{A ; B ; C ; D}\left(\rho^{A B C D}\right)=S\left(\rho^{A B}\right)-S\left(\rho^{\prime A}\right)+$ $S\left(\rho^{\prime \prime A B C}\right)-S\left(\rho^{\prime \prime A B}\right)+S\left(\rho^{\prime \prime \prime A B C D}\right)-S\left(\rho^{\prime \prime A B C}\right)-S\left(\rho^{A B C D}\right)+S\left(\rho^{A}\right)$ for some $\Pi^{A B C}$ with $\Pi^{A}\left(\rho^{A B C D}\right)=\rho^{A B C D}, \Pi^{A B}\left(\rho^{A B C D}\right)=\rho^{\prime \prime A B C D}$ and $\Pi^{A B C}\left(\rho^{A B C D}\right)=\rho^{\prime \prime \prime A B C D}$. (Hereafter, in this subsection, we denote by $\rho^{\prime}$ the state after the optimal local measurement on one particle of the four-partite state, by $\rho^{\prime \prime}$ the state after the optimal local measurement on two particles of the four-partite state, and by $\rho^{\prime \prime \prime}$ the state after the optimal local measurement on three particles of the four-partite state.) Obviously, $D_{A ; B ; C}\left(\rho^{A B C}\right) \leq S_{A B}^{\prime}-S_{A}^{\prime}+S_{A B C}^{\prime \prime}-S_{A B}^{\prime \prime}-S_{B C}+S^{A}$ and $S_{A B C D}^{\prime \prime \prime}-S_{A B C}^{\prime \prime \prime}-S_{A B C D}+S_{A B C} \geq$ $D_{A B C ; D}$. It is straightforward that

$$
\begin{aligned}
& D_{A ; B ; C: D}-D_{A ; B ; C} \\
\geq & \left(-S_{A B C D}+S_{A}+S_{A B}^{\prime}-S_{A}^{\prime}+S_{A B C}^{\prime \prime}-S_{A B}^{\prime \prime}+S_{A B C D}^{\prime \prime \prime}-S_{A B C}^{\prime \prime \prime}\right) \\
& -\left(S_{A B}^{\prime}-S_{A}^{\prime}+S_{A B C}^{\prime \prime}-S_{A B}^{\prime \prime}-S_{A B C}+S^{A}\right) \\
= & \left(S_{A B C D}^{\prime \prime \prime}-S_{A B C}^{\prime \prime \prime}-S_{A B C D}+S_{A B C}\right)+\left(S_{A B C}^{\prime \prime}-S_{A B}^{\prime \prime}-S_{A B C}+S_{A B}\right) \\
& +\left(S_{A B}^{\prime}-S_{A}^{\prime}-S_{A B}+S_{A}\right)-\left(S_{A B C}^{\prime \prime}-S_{A B}^{\prime \prime}-S_{A B C}+S_{A B}\right) \\
& -\left(S_{A B}^{\prime}-S_{A}^{\prime}-S_{A B}+S_{A}\right) \\
= & S_{A B C D}^{\prime \prime \prime}-S_{A B C}^{\prime \prime \prime}-S_{A B C D}+S_{A B C} \\
\geq & D_{A B C ; D}
\end{aligned}
$$

and

$$
\begin{aligned}
& D_{A ; B ; C: D}-D_{A ; B ; D} \\
\geq & \left(S_{A B C D}^{\prime \prime \prime}-S_{A B C}^{\prime \prime \prime}-S_{A B C D}+S_{A B C}\right)+\left(S_{A B C}^{\prime \prime}-S_{A B}^{\prime \prime}-S_{A B C}+S_{A B}\right)
\end{aligned}
$$


Monogamy of quantum discord

$$
\begin{aligned}
& +\left(S_{A B}^{\prime}-S_{A}^{\prime}-S_{A B}+S_{A}\right)-\left(S_{A B D}^{\prime \prime}-S_{A B}^{\prime \prime}-S_{A B D}+S_{A B}\right) \\
& -\left(S_{A B}^{\prime}-S_{A}^{\prime}-S_{A B}+S_{A}\right) \\
= & \left(S_{A B C D}^{\prime \prime \prime}-S_{A B C}^{\prime \prime \prime}-S_{A B C D}+S_{A B C}\right)-\left(S_{A B D}^{\prime \prime}-S_{A B}^{\prime \prime}-S_{A B D}+S_{A B}\right) \\
& +\left(S_{A B C}^{\prime \prime}-S_{A B}^{\prime \prime}-S_{A B C}+S_{A B}\right) \\
= & \left(S_{A B C D}^{\prime \prime \prime}-S_{A B C}^{\prime \prime \prime}-S_{A B C D}^{\prime \prime}+S_{A B C}^{\prime \prime}\right) \\
& +\left(S_{A B C D}^{\prime \prime}-S_{A B D}^{\prime \prime}-S_{A B C D}+S_{A B D}\right) \\
\geq & 0 .
\end{aligned}
$$

Similarly,

$$
\begin{aligned}
& D_{A ; B ; C: D}-D_{A ; C ; D} \\
\geq & \left(S_{A B C D}^{\prime \prime \prime}-S_{A B C}^{\prime \prime \prime}-S_{A B C D}+S_{A B C}\right)+\left(S_{A B C}^{\prime \prime}-S_{A B}^{\prime \prime}-S_{A B C}+S_{A B}\right) \\
& +\left(S_{A B}^{\prime}-S_{A}^{\prime}-S_{A B}+S_{A}\right)-\left(S_{A C D}^{\prime \prime}-S_{A C}^{\prime \prime}-S_{A C D}+S_{A C}\right) \\
& -\left(S_{A C}^{\prime}-S_{A}^{\prime}-S_{A C}+S_{A}\right) \\
= & \left(S_{A B C D}^{\prime \prime \prime}-S_{A B C}^{\prime \prime \prime}-S_{A B C D}+S_{A B C}\right)-\left(S_{A C D}^{\prime \prime}-S_{A C}^{\prime \prime}-S_{A C D}+S_{A C}\right) \\
& +\left(S_{A B C}^{\prime \prime \prime}-S_{A B}^{\prime \prime \prime}-S_{A B C}+S_{A B}\right)+\left(S_{A B}^{\prime}-S_{A}^{\prime}-S_{A B}+S_{A}\right) \\
& -\left(S_{A C}^{\prime}-S_{A}^{\prime}-S_{A C}+S_{A}\right) \\
= & \left(S_{A B C D}^{\prime \prime \prime}-S_{A B C}^{\prime \prime \prime}-S_{A B C D}+S_{A B C}\right)-\left(S_{A C D}^{\prime \prime}-S_{A C}^{\prime \prime}-S_{A C D}+S_{A C}\right) \\
& +\left(S_{A B C}^{\prime \prime}-S_{A B}^{\prime \prime \prime}-S_{A B C}^{\prime}+S_{A B}^{\prime}\right)+\left(S_{A B C}^{\prime}-S_{A C}^{\prime}-S_{A B C}+S_{A C}\right) \\
\geq & \left(S_{A B C D}^{\prime \prime \prime}-S_{A B C}^{\prime \prime \prime}-S_{A B C D}+S_{A B C}\right)-\left(S_{A C D}^{\prime \prime}-S_{A C}^{\prime \prime}-S_{A C D}+S_{A C}\right) \\
\geq & 0
\end{aligned}
$$

provided that $d_{A B C ; D} \geq d_{A C ; D}$. Evidently, $D_{A ; B ; C: D}-D_{A ; C ; D} \geq D_{A ; B}$ whenever $d_{A B C ; D} \geq d_{A C ; D}$ and $d_{A B ; C} \geq d_{A ; C}$. With the same argument, one can check that $D_{A ; B ; C ; D} \geq D_{B ; C ; D}+D_{A ; B}$ provided that $d_{A B C ; D} \geq d_{B C ; D}$ and $d_{A B ; C} \geq d_{B ; C}$. Since $D_{A ; B ; C ; D} \geq D_{A ; B ; C}$ and $D_{A ; B ; C} \geq D_{A ; B}, D_{A ; B ; C} \geq D_{A ; C}$, we get $D_{A ; B ; C ; D} \geq D_{A ; B}$ and $D_{A ; B ; C ; D} \geq D_{A ; C}$ directly. We also have $D_{A ; B ; C: D} \geq D_{A ; D}$ since

$$
\begin{aligned}
& D_{A ; B ; C: D}-D_{A ; D} \\
\geq & \left(S_{A B C D}^{\prime \prime \prime}-S_{A B C}^{\prime \prime \prime}-S_{A B C D}+S_{A B C}\right)+\left(S_{A B C}^{\prime \prime}-S_{A B}^{\prime \prime}-S_{A B C}+S_{A B}\right) \\
& +\left(S_{A B}^{\prime}-S_{A}^{\prime}-S_{A B}+S_{A}\right)-\left(S_{A D}^{\prime}-S_{A}^{\prime}-S_{A D}+S_{A}\right) \\
= & \left(S_{A B C D}^{\prime \prime \prime}-S_{A B C}^{\prime \prime \prime}-S_{A B C D}^{\prime}+S_{A B C}^{\prime}\right)+\left(S_{A B C D}^{\prime}-S_{A D}^{\prime}-S_{A B C D}+S_{A D}\right) \\
& +\left(S_{A B C}^{\prime \prime}-S_{A B}^{\prime \prime}-S_{A B C}^{\prime}+S_{A B}^{\prime}\right) \\
\geq & 0 .
\end{aligned}
$$

The other items can also be easily checked, and thus the proof is completed.

Proposition 5. $D_{A ; B ; C ; D}$ is completely monogamous if it is complete, i.e.,

- $d_{A B C ; D} \geq d_{X Y ; D}$ for $\{X, Y\} \in\{\{A, C\},\{B, C\}\}$.

- $d_{A B C ; D} \geq d_{Z ; D}$ for $Z \in\{A, B$,$\} .$

- $d_{A B ; C} \geq d_{B ; C}$ 
hold for any state $\rho \in \mathcal{S}^{A B C D}$.

Proof. For convenience, we use the same notations as that of the proof of Proposition 4. If $D_{A ; B ; C ; D}\left(\rho^{A B C D}\right)=D_{A ; B}\left(\rho^{A B}\right)$, then $S_{A B C D}^{\prime \prime \prime}-S_{A B C}^{\prime \prime \prime}-S_{A B C D}+S_{A B C}=0$ and $S_{A B C}^{\prime}-S_{A B}^{\prime}-S_{A B C}+S_{A B}=0$, which implies that $\rho^{A B C D}=\sum_{k, j, l} p_{k, j, l}|k\rangle\left\langle\left. k\right|^{A} \otimes\right.$ $|j\rangle\left\langle\left. j\right|^{B} \otimes \mid l\right\rangle\left\langle\left. l\right|^{C} \otimes \rho_{k, j, l}^{D}\right.$. Thus $D_{A ; C}=D_{A ; D}=D_{B ; C}=D_{B ; D}=D_{C ; D}=0$. If $D_{A ; B ; C ; D}\left(\rho^{A B C D}\right)=D_{A ; B ; C}\left(\rho^{A B C}\right)$, then $S_{A B C D}^{\prime \prime \prime}-S_{A B C}^{\prime \prime \prime}-S_{A B C D}+S_{A B C}=0$, which implies that $\rho^{A B C D}=\sum_{k, j, l} p_{k, j, l}|k\rangle\left\langle\left. k\right|^{A} \otimes \mid j\right\rangle\left\langle\left. j\right|^{B} \otimes \mid l\right\rangle\left\langle\left. l\right|^{C} \otimes \rho_{k, j, l}^{D}\right.$. Thus $D_{A ; D}=D_{B ; D}=$ $D_{C ; D}=0$. Similarly, one can easily verify that all the other dis-correlate conditions are valid.

\subsection{The n-partite case}

Moving to general $n$-partite system, we can conclude the following theorems which are main results of this paper by similar arguments as that of Propositions 4 and 5 .

Theorem 6. Let $D_{A_{1} ; A_{2} ; \cdots ; A_{n}}$ be the n-partite quantum discord defined on $\mathcal{S}^{A_{1} A_{2} \cdots A_{n}}$. Then the following holds true for any state $\rho \in \mathcal{S}^{A_{1} A_{2} \cdots A_{n}}$ :

- $D_{A_{1} ; A_{2} ; \cdots ; A_{n}} \geq D_{A_{1} A_{2} \cdots A_{n-1} ; A_{n}}+D_{A_{1} ; A_{2} ; \cdots ; A_{n-1}}$.

- $D_{A_{1} ; A_{2} ; \cdots ; A_{n}} \geq D_{A_{1} ; A_{2} ; \cdots ; A_{p} ; A_{3}}, 2 \leq p \leq n-1$.

- $D_{A_{1} ; A_{2} ; \cdots ; A_{n}} \geq D_{A_{1} ; A_{i}}, 3 \leq i \leq n$.

- $D_{X_{1} ; X_{2} ; \cdots ; X_{k}} \geq D_{Y_{1} ; Y_{2} ; \cdots ; Y_{l}}$ for any $X_{1}\left|X_{2}\right| \cdots\left|X_{k} \succ^{b} Y_{1}\right| Y_{2}|\cdots| Y_{l}$. Moreover, $D_{X_{1} ; X_{2} ; \cdots ; X_{k}} \geq D_{Y_{1} ; Y_{2} ; \cdots ; Y_{l}}+D_{X_{1} ; X_{2} ; \cdots ; X_{q}}$ whenever $X_{1}\left|X_{2}\right| \cdots\left|X_{k} \succ^{b} Y_{1}\right| Y_{2}|\cdots| Y_{l}$ with $Y_{1}=X_{1} X_{2} \cdots X_{q}$.

- If

$$
d_{Z_{1} Z_{2} \cdots Z_{s} ; Z_{s+1}} \geq d_{Z_{i_{1}} Z_{i_{2}} \cdots Z_{i_{t}} ; Z_{s+1}}
$$

holds for any $Z_{1}\left|Z_{2}\right| \cdots\left|Z_{s} \succ^{a} Z_{i_{1}}\right| Z_{i_{2}}|\cdots| Z_{i_{t}}$, then

$$
D_{X_{1} ; X_{2} ; \cdots ; X_{k}} \geq D_{Y_{1} ; Y_{2} ; \cdots ; Y_{l}}
$$

for any $X_{1}\left|X_{2}\right| \cdots\left|X_{k} \succ^{a} \quad Y_{1}\right| Y_{2}|\cdots| Y_{l}$, where $Z_{1}\left|Z_{2}\right| \cdots\left|Z_{s}, \quad Z_{i_{1}}\right| Z_{i_{2}}|\cdots| Z_{i_{t}}$, $X_{1}\left|X_{2}\right| \cdots \mid X_{k}$ and $Y_{1}\left|Y_{2}\right| \cdots \mid Y_{l}$ are partitions of $A_{1} A_{2} \cdots A_{n}$ or subsystem of $A_{1} A_{2} \cdots A_{n}$.

That is, if Eq. (30) holds true, then $D_{A_{1} ; A_{2} ; \cdots ; A_{n}}$ is a complete multiparty quantum discord.

Proof. For any $n$, analogous to that of tripartite and four-partite cases, all these coarsening relations can be derived by taking the optimal measurement for the former one [e.g., in relation $D_{A ; B ; C ; D} \geq D_{A ; B C D}$, we assume that $D_{A ; B ; C ; D}$ is obtained by the optimal measurement, i.e., $D_{A ; B ; C ; D}=-S_{A B C D}+S_{A}+S_{A B}^{\prime}-S_{A}^{\prime}+S_{A B C}^{\prime \prime}-S_{A B}^{\prime \prime}+S_{A B C D}^{\prime \prime \prime}-$ $S_{A B C}^{\prime \prime \prime}=\left(S_{A B C D}^{\prime \prime \prime}-S_{A B C}^{\prime \prime \prime}-S_{A B C D}+S_{A B C}\right)+\left(S_{A B C}^{\prime \prime}-S_{A B}^{\prime \prime}-S_{A B C}+S_{A B}\right)+\left(S_{A B}^{\prime}-S_{A}^{\prime}-\right.$ $\left.S_{A B}+S_{A}\right)$ ], and then by using either the fact that the mutual information is decreased under local operation (which is equivalent to $S_{X Y}^{\prime}-S_{X}^{\prime}-S_{X Y}+S_{X} \geq 0$ ), or such a 
fact togeter with the assumption (30). The former four cases can be derived without the assumption (30) since all these cases can be reduced to $S_{X Y}^{\prime}-S_{X}^{\prime}-S_{X Y}+S_{X} \geq 0$. The last case can be easily processed under the assumption (30).

Using similar arguments as that of Proposition 2 and Proposition 5, we can conclude the following theorem easily.

Theorem 7. The multipartite discord $D_{A_{1} ; A_{2} ; \cdots ; A_{n}}$ is completely monogamous if it is complete, or equivalently, if it is monotonic under coarsening of subsystem(s),.

The former four monotonicity relations in Theorem 6 are true automatically. So, in Theorem 7, we only need the other monotonicity conditions as Eq. (31). (Here, we should note that Eq. (31) contains the second and the third monotonicity relations in Theorem 6 as special cases.) We thus obtain the following proposition.

Proposition 8. The multipartite discord $D_{A_{1} ; A_{2} ; \cdots ; A_{n}}$ is completely monogamous provided that the condition (31) is valid.

Although $D_{A_{1} ; A_{2} ; \cdots ; A_{n}}$ is not continuous (since $D_{A_{1} ; A_{2}}$ is not continuous $[6,32]$ ), $n>2$, we still can, taking the tripartite case for example, get the following monogamy relation: If $D_{A ; B ; C}$ is monotonic under discord of subsystems, then for any given $\rho \in \mathcal{S}^{A B C}$ there exists $0<\alpha<\infty$ such that

$$
D_{A ; B ; C}^{\alpha} \geq D_{A ; B}^{\alpha}+D_{A ; C}^{\alpha}+D_{B ; C}^{\alpha} .
$$

That is, $\alpha$ is dependent not only on $\operatorname{dim} \mathcal{H}^{A B C}$ but also on the given state.

\section{The coarser relation of global quantum discord}

In Ref. [23], the following monogamy bound is proved provided that the bipartite discord does not increase under loss of subsystems, i.e., $D_{A_{1} \cdots A_{k}: A_{k+1}} \geq D_{A_{1}: A_{k+1}}, 2 \leq k<n$ :

$$
D_{A_{1}: \cdots: A_{n}} \geq \sum_{k=1}^{n-1} D_{A_{1}: A_{k+1}} .
$$

However, the assumption $D_{A_{1} \cdots A_{k}: A_{k+1}} \geq D_{A_{1}: A_{k+1}}$ is not valid in general. For example, consider a three qubit state $\rho_{A B C}=(1 / 2)(|000\rangle\langle 000|+|+11\rangle\langle+11|)$, where $|+\rangle=$ $(|0\rangle+|1\rangle) / \sqrt{2}$, it is evident that $D_{A B: C}=0<D_{A: C}$. That is, $D_{A_{1} \cdots A_{k}: A_{k+1}}$ and $D_{A_{1}: A_{k+1}}$ are uncomparable since the associated measurements are not compatible.

For any state $\rho \in \mathcal{S}^{A B C}$, we let $D_{A: B: C}(\rho)=S_{A B C}^{\prime}-S_{A}^{\prime}-S_{B}^{\prime}-S_{C}^{\prime}-S_{A B C}+S_{A}+$ $S_{B}+S_{C}$ for some local von Neumann measurement $\Phi(\rho)=\rho^{\prime}$. It follows that

$$
\begin{aligned}
D_{A: B: C}-D_{A: B C} \geq & \left(S_{A B C}^{\prime}-S_{A}^{\prime}-S_{B}^{\prime}-S_{C}^{\prime}-S_{A B C}+S_{A}+S_{B}+S_{C}\right) \\
& -\left(S_{A B C}^{\prime}-S_{A}^{\prime}-S_{B C}^{\prime}-S_{A B C}+S_{A}+S_{B C}\right) \\
= & \left(S_{B}+S_{C}-S_{B C}\right)-\left(S_{B}^{\prime}+S_{C}^{\prime}-S_{B C}^{\prime}\right) \geq 0
\end{aligned}
$$

since mutual information is decreased under local von Neumann measurement. With the notations as in Section 4, we let $X_{1}\left|X_{2}\right| \cdots \mid X_{k}$ and $Y_{1}\left|Y_{2}\right| \cdots \mid Y_{l}$ be two arbitrarily 
given partitions of $A_{1} A_{2} \cdots A_{m}$ or subsystem of $A_{1} A_{2} \cdots A_{m}$, and assume that $X_{1}\left|X_{2}\right| \cdots\left|X_{k} \succ^{b} Y_{1}\right| Y_{2}|\cdots| Y_{l}$. Then we can conclude $D_{X_{1}: X_{2}: \cdots: X_{k}} \geq D_{Y_{1}: Y_{2}: \cdots: Y_{l}}$.

We now begin to discuss the coarsening relation of GQD as a complement to the results in Ref. [23]. For any state $\rho^{X Y} \in \mathcal{S}^{X Y}$, we denote $S\left(\rho^{X Y} \| \rho^{X} \otimes \rho^{Y}\right)$ by $S_{X Y \| X \otimes Y}$ for brevity, where $S(\rho \| \sigma)=\operatorname{tr}\left(\rho \log _{2} \rho-\rho \log _{2} \sigma\right)$ is the relative entropy. For any state $\rho \in \mathcal{S}^{A_{1} A_{2} \cdots A_{n}}$, we write state after measurement $\Phi$ as in Eq. (5) as $\rho^{\prime}$, write $S\left(\rho^{\prime X Y} \| \rho^{\prime X} \otimes \rho^{\prime Y}\right)$ as $S_{X Y \| X \otimes Y}^{\prime}$, and write $I(\rho)-I\left(\rho^{\prime}\right)$ as $d_{A_{1}: A_{2}: \cdots: A_{n}}^{\Phi}$. Then, it is easy to argue that, for any $k<n$,

$$
\begin{aligned}
& d_{A_{1}: A_{2}: \cdots: A_{n}}^{\Phi}-d_{A_{i_{1}}: A_{i_{2}}: \cdots: A_{i_{k}}}^{\Phi} \\
= & S_{A_{1} A_{2} \cdots A_{n} \| A_{i_{1}} A_{i_{2}} \cdots A_{i_{k}} \otimes A_{j_{1}} \otimes A_{j_{2}} \otimes \cdots \otimes A_{j_{n-k}}} \\
& -S_{A_{1} A_{2} \cdots A_{n} \| A_{i_{1}} A_{i_{2}} \cdots A_{i_{k}} \otimes A_{j_{1}} \otimes A_{j_{2}} \otimes \cdots \otimes A_{j_{n-k}}}^{\prime} .
\end{aligned}
$$

where $j_{s} \neq i_{t}, 1 \leq t \leq k, 1 \leq j \leq n-k$. With these notations in mind, we can easily conclude the following coarsening relations.

Proposition 9. (i) Using the notations as Eq. (34), if $d_{A_{1}: A_{2}: \cdots: A_{n}}^{\Phi} \geq d_{A_{i_{1}}: A_{i_{2}}: \cdots: A_{i_{k}}}^{\Phi}$ for any $k<n$, then $D_{A_{1}: A_{2}: \cdots: A_{n}}$ is monotonic under discord of subsystems. (ii) $D_{X_{1}: X_{2}: \cdots: X_{k}} \geq D_{Y_{1}: Y_{2}: \cdots: Y_{l}}$ whenever $X_{1}\left|X_{2}\right| \cdots\left|X_{k} \succ^{b} Y_{1}\right| Y_{2}|\cdots| Y_{l}$. Namely, $D_{A_{1}: A_{2}: \cdots: A_{n}}$ is complete whenever $d_{A_{1}: A_{2}: \cdots: A_{n}}^{\Phi} \geq d_{A_{i_{1}}: A_{i_{2}}: \cdots: A_{i_{k}}}^{\Phi}$ for any $k<n$.

Next, we consider the complete monogamy of $D_{A_{1}: A_{2}: \cdots: A_{n}}$. Take a three qubit state

$\rho_{A B C}=(1 / 2)(|000\rangle\langle 000|+| 1+1\rangle\langle 1+1|)$ as in Ref. [23], where $|+\rangle=(|0\rangle+|1\rangle) / \sqrt{2}$. For this state, it is shown in Ref. [23] that $D_{A: B: C}=D_{A: B} \approx 0.204$ and $D_{A: C}=0$. It is easy to see that $D_{B: C}=D_{A: B}$. That is, $D_{A: B: C}=D_{A: B}$ but $D_{B: C} \neq 0$. Namely, GQD violates the dis-correlated condition. We thus obtain the following theorem.

Theorem 10. $D_{A_{1}: \cdots: A_{n}}$ is not completely monogamous.

That is, even though GQD is complete, it is not completely monogamous. Comparing with MQD, MQD $D_{A_{1} ; A_{2} ; \cdots ; A_{n}}$ is better than GQD $D_{A_{1}: A_{2}: \cdots: A_{n}}$ in such a sense. Of course that, $D_{A_{1}: A_{2}: \cdots: A_{n}}$ has some merit, e.g., it is symmetric but $D_{A_{1} ; A_{2} ; \cdots ; A_{n}}$ is not symmetric (see Fig. 1 for more detail).

\section{Conclusions and discussions}

In this paper we have discussed the monogamy relation of multipartite quantum discord and global quantum discord in detail. Different from the monogamy scenario of quantum discord discussed in the previous literatures, we defined complete multiparty quantum discord and by which we put forward the complete monogamy framework for both multipartite quantum discord and global quantum discord. In such a framework, to characterize the distribution of quantum discord, the first issue is to check whether the quantity is monotonic under coarsening of subsystems and the other issue it to investigate the dis-correlate condition. The dis-correlate condition of quantum discord is the counterpart to the disentanglement condition for entanglement. With the assumption of monotonicity under coarsening of subsystems, 
Table 1. Comparison between MQD, GQD and complete multipartite entanglement measure for tripartite case. CM is the abbreviation of 'complete monogamous'.

\begin{tabular}{lcccccc}
\hline Measure & U1 & U2 & U3 & U4 & CM \\
\hline$D_{A_{1} ; A_{2} ; A_{3}}$ & $\checkmark$ & $\times$ & Partially, conditionally $^{a}$ & $\checkmark$ & Conditionally $^{b}$ \\
$D_{A_{1}: A_{2}: A_{3}}$ & $\checkmark$ & $\checkmark$ & Conditionally $^{c}$ & $\checkmark$ & $\times$ \\
$E_{f}^{(3)}$ & $\checkmark$ & $\checkmark$ & $\checkmark$ & $\checkmark$ & $\checkmark$ \\
\hline${ }^{a}$ See Theorem 6. & & & & \\
${ }^{b}$ See Theorem 7. \\
${ }^{c}$ See Proposition 9.
\end{tabular}

we proved that the multipartite quantum discord is completely monogamous. We presented counterexamples which implies that global quantum discord is not completely monogamous. This fact also supports that the multipartite quantum discord in Ref. [11] is an excellent generalization of quantum discord. Going further, we conjecture that the assumptions in Theorem 6, Theorem 7, Proposition 8 and Proposition 9 are true but it seems difficult to prove and remains further investigation in the future. In addition, our framework can be used for any multiparty nonlocal quantum correlation whenever the associated monogamy relation is concerned.

\section{Acknowledgments}

This work is supported by the National Natural Science Foundation of China under Grant No. 11971277, the Program for the Outstanding Innovative Teams of Higher Learning Institutions of Shanxi, the Scientific Innovation Foundation of the Higher Education Institutions of Shanxi Province under Grants No. 2019KJ034, No. 2019L0742, and No. 2020L0471, the Natural Science Foundation of Shanxi Province under Grant No. 201801D121016, and the Science Technology Plan Project of Datong City, China under Grants No. 2018151 and No. 2020153.

\section{References}

[1] Ollivier H and Zurek W H 2001 Quantum discord: a measure of the quantumness of correlations Phys. Rev. Lett. 88017901

[2] Henderson L and Vedral V 2001 Classical, quantum and total correlations J. Phys. A: Math. Theor. 346899

[3] Datta A, Shaji A, and Caves C M 2008 Quantum discord and the power of one qubit Phys. Rev. Lett. 100050502

[4] Datta A and Gharibian S 2009 Signatures of nonclassicality in mixed-state quantum computation Phys. Rev. A 79042325

[5] Sarandy M S 2009 Classical correlation and quantum discord in critical systems. Phys. Rev. A 80 022108

[6] Xu J S, Xu X Y, Li C F et al 2010 Experimental investigation of classical and quantum correlations under decoherence Nat. Commun. 17

[7] Rulli C C and Sarandy M S 2011 Global quantum discord in multipartite systems Phys. Rev. A 84042109 
[8] Giorgi G L, Bellomo B, Galve F et al 2011 Genuine quantum and classical correlations in multipartite Systems Phys. Rev. Lett. 107190501

[9] Modi K, Brodutch A, Cable H et al 2012 The classical-quantum boundary for correlations: Discord and related measures Rev. Mod. Phys. 841655

[10] Bera A, Das T, Sadhukhan D et al 2018 Quantum discord and its allies: a review Rep. Prog. Phys. 81024001

[11] Radhakrishnan C, Laurière M and Byrnes T 2020 Multipartite generalization of quantum discord Phys. Rev. Lett. 124110401

[12] Hu M L, Hu X, Wang J et al 2018 Quantum coherence and geometric quantum discord Phys. Rep. 7621

[13] Zhou H, Yuan X, Ma X 2019 Unification of quantum resources in distributed scenarios Phys. Rev. A 99022326

[14] Coffman V, Kundu J and Wootters W K 2000 Distributed entanglement Phys. Rev. A 61052306

[15] Streltsov A, Adesso G, Piani M et al 2012 Are general quantum correlations monogamous? Phys. Rev. Lett. 109050503

[16] Dhar H S, Pal A K, Rakshit D et al 2017 Monogamy of quantum correlations-a review In Lectures on General Quantum Correlations and their Applications(Cham, Switzerland: Springer)

[17] Gour G and Guo Y 2018 Monogamy of entanglement without inequalities Quantum 281

[18] Guo Y and Gour G 2019 Monogamy of the entanglement of formation Phys. Rev. A 99042305

[19] Guo Y and Zhang L 2020 Multipartite entanglement measure and complete monogamy relation Phys. Rev. A 101032301

[20] Guo Y, Zhang L and Yuan H 2020 Entanglement measures induced by fidelity-based distances Quant. Inf. Process. 19282

[21] Eltschka C and Siewert J 2018 Distribution of entanglement and correlations in all finite dimensions Quantum 264

[22] Deng X, Xiang Y, Tian C et al 2017 Demonstration of monogamy relations for Einstein-PodolskyRosen steering in Gaussian cluster state Phys. Rev. Lett. 118230501

[23] Braga H C, Rulli C C, Oliveira T R de et al 2012 Monogamy of quantum discord by multipartite correlations Phys. Rev. A $\mathbf{8 6} 062106$

[24] Ren X J and Fan H 2013 Non-monogamy of quantum discord and upper bounds for quantum correlation Quant. Inf. Comput. 13(5-6) 0469-0478

[25] Prabhu R, Pati A K, SenDe A et al 2012 Conditions for monogamy of quantum correlations: Greenberger-Horne-Zeilinger versus W states Phys. Rev. A 85 040102(R)

[26] Giorgi G L 2011 Monogamy properties of quantum and classical correlations Phys. Rev. A 84 054301

[27] Fanchini F F et al 2013 Why the Entanglement of Formation is not generally monogamic Phys. Rev. A 87032317

[28] Bai Y K et al 2013 Exploring multipartite quantum correlations with the square of quantum discord Phys. Rev. A $\mathbf{8 8} 012123$

[29] Groisman B, Popescu S and Winter A 2005 Quantum, classical, and total amount of correlations in a quantum state Phys. Rev. A $\mathbf{7 2} 032317$

[30] Horodecki R, Horodecki P, Horodecki M and Horodecki K 2009 Quantum entanglement Rev. Mod. Phys. 81865

[31] Nielsen M A, Chuang I L 2000 Quantum Computatation and Quantum Information (Cambridge: Cambridge University Press)

[32] Mazzola L, Piilo J and Maniscalco S 2010 Sudden transition between classical and quantum decoherence Phys. Rev. Lett. 104200401 